\title{
Effect of Thickness of Electroplated NiFe Cores on the Noise of Fluxgates
}

\begin{abstract}
M. ButTA*
Department of Measurement, Faculty of Electrical Engineering, Czech Technical University in Prague, 16627 Prague, Czech Republic

In this paper we present the effect of the thickness on the field-induced anisotropy of NiFe layers. We electroplated several ring-cores with thickness spanning from $2 \mu \mathrm{m}$ to $18 \mu \mathrm{m}$ changing the deposition time (and keeping unchanged the other parameters). The ring-cores were placed during the electroplating process in yoke, which produced $720 \mathrm{kA} / \mathrm{m}$ radial field in order to induce a radial anisotropy to the ring. We observed that the field-induced anisotropy significantly increases up to $12 \mu \mathrm{m}$ thickness; for further increment of thickness the anisotropy does not increase anymore. The effect is visible on the noise of the fluxgate based on such rings: the noise quickly drops for increase of thickness due to larger radial anisotropy. However, for $18 \mu \mathrm{m}$ the noise slightly rises due to emerging of demagnetizing effect. Therefore we derive that $12 \mu \mathrm{m}$ is the best trade-off between the need of larger field-induced anisotropy and low demagnetization.
\end{abstract}

DOI: 10.12693/APhysPolA.131.756

PACS/topics: 07.55.Ge, 75.30.Gw, 75.50.Bb

\section{Introduction}

The geometry of the core plays a crucial role in a fluxgate sensor. A common rule usually followed when designing a fluxgate sensor is to keep the thickness of the core very small. The main reason behind it is the need to minimized the effect of the demagnetizing factor [1] to the measured magnetic field, and thus maximize the sensitivity.

A typical ring core of a fluxgate is indeed exposed to two different fields: the excitation field and the measured field (Fig. 1). With the respect of the excitation field the ring appears to be a ring exposed to a circular field, therefore it shows no demagnetizing effect. However, from the point of view of the measured field, the ring is affected by demagnetizing effect. While for race-track cores [2] it is possible to approximate the sensitive sections of the core as parallelepiped and approximate the calculus of the demagnetizing factor, for ring cores this is in fact very difficult due to the fact that the sensitive area has not constant cross section. In any case, thinner core corresponds always to lower demagnetizing effect, therefore with higher coupling of the ferromagnetic core to the measured field.

The final sensitivity of the sensor is eventually given by the tradeoff between the need of low demagnetization effect obtained at low thickness and the need of high flux obtained increasing the ferromagnetic material crosssection obtained with larger thickness.

However, this simple criterion becomes more complicated when other thickness-dependent parameters have to be taken into account.

*corresponding author; e-mail: buttamat@fel.cvut.cz
We have previously shown [3] that radial anisotropy induced in the ring by exposing it to radial field during the electroplating is an effective method to reduce the noise of electroplated $\mathrm{NiFe}$ rings for fluxgates. In this paper we study how such field-induced anisotropy is affected by the thickness of the layer and eventually how this influences the noise of the sensor.

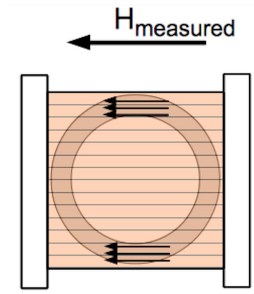

a)

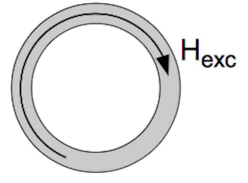

b)

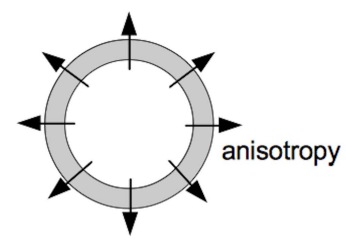

c)

Fig. 1. a) Measured field with the respect of the ring (affected by demagnetization) - b) Excitation field $H_{\text {exc }}$ not affected by demagnetization - c) Direction of radial anisotropy desired to reduce noise.

\section{Manufacturing of the cores}

We manufactured several cores electroplating NiFe alloy on $9 \mu \mathrm{m}$ thick copper layer over a $250 \mu \mathrm{m}$ thick fiberglass substrate. The electroplating we performed using a solution of $\mathrm{FeSO}_{4} \cdot 7 \mathrm{H}_{2} \mathrm{O}(8 \mathrm{~g} / \mathrm{l}), \mathrm{NiSO}_{4} \cdot 6 \mathrm{H}_{2} \mathrm{O}$ 
(125 g/l), $\mathrm{NiCl}_{2} \cdot 6 \mathrm{H}_{2} \mathrm{O}(20 \mathrm{~g} / \mathrm{l}), \mathrm{H}_{3} \mathrm{BO}_{3}(40 \mathrm{~g} / \mathrm{l})$, saccharin $(6 \mathrm{~g} / \mathrm{l})$ in de-mineralized water at $55^{\circ} \mathrm{C}$ with $\mathrm{pH}$ corrected to 2.8 adding $\mathrm{KOH}[4]$. The electroplating current was at $16 \mathrm{~mA} / \mathrm{cm}^{2}$ current density, which provides a classical $81: 19 \mathrm{Ni}-\mathrm{Fe}$ ratio, which has been proven to give the lowest magnetostriction (ideal condition for low noise). The ring is exposed to a $720 \mathrm{kA} / \mathrm{m}$ radial field produced by a yoke. This will induce a radial anisotropy which reduces the Barkhausen noise in the core and therefore the final noise of the sensor [5]. The external diameter of the ring is $47 \mathrm{~mm}$ and the internal diameter is $41 \mathrm{~mm}$, giving $3 \mathrm{~mm}$ wide track.

The time of electroplating was changed in order to modify the thickness of the resulting layer. The shortest time was $10 \mathrm{~min}$, corresponding to $2 \mu \mathrm{m} \mathrm{NiFe} \mathrm{layer.} \mathrm{Hig-}$ her times of electroplating were 20,30, 45, 60, and $90 \mathrm{~min}$ corresponding to $4,6,9,12$, and $18 \mu \mathrm{m}$. The thickness has been estimated by previous characterization by surface profiler of the dependence of the thickness on the electroplating time for a given current density.

The rings have been electroplated in groups of three cores for every bath. After the electrodeposition of three cores a new bath was prepared in order to assure a uniform stoichiometry of the components. We manufactured four cores for each thickness and by doing so we assured to periodically change the order of electroplating, in order to avoid any possible effect of bath change which might affect the last electroplated rings.

\section{Field-induced anisotropy}

The different thickness has a strong effect on the field induced anisotropy. Figure 2 shows the circular $B-H$ loops of the two rings, with $2 \mu \mathrm{m}$ and $12 \mu \mathrm{m}$ thickness, measured with traditional induction method at $100 \mathrm{~Hz}$. The harder the loop appears the higher is the anisotropy in radial direction. The field-induced anisotropy is uniform all over the circumference of the ring.

We can see that the larger is the thickness, the larger is the field anisotropy. While at $2 \mu \mathrm{m}$ the effect of the radial field can hardly be observed, at $12 \mu \mathrm{m}$ the field-induced anisotropy becomes clear. In Fig. 2 we have not shown for sake of clarity the $B-H$ loops of the other rings. However, the anisotropy increases from $2 \mu \mathrm{m}$ thickness to $12 \mu \mathrm{m}$. Then, for larger thickness (namely $18 \mu \mathrm{m}$ ) the $B-H$ loop appears to be the same as for $12 \mu \mathrm{m}$. This indicates that the field-induced radial anisotropy increases only up to $12 \mu \mathrm{m}$ where it reaches its maximum.

It should be noted also that the $B-H$ loop of thick cores has still a central part which appears to have large anisotropy and high permeability, while the loop gets slimmer while it reaches the saturation. This indicates that the ring is composed of different sections: the first one with low radial anisotropy (corresponding to the first $\mu \mathrm{m}$, where the effect of the field is still low) and the following electrodeposited material with larger radial anisotropy. Therefore what we see in Fig. 2 is the overlapping of a low and high radial anisotropy behaviors in a single ring.

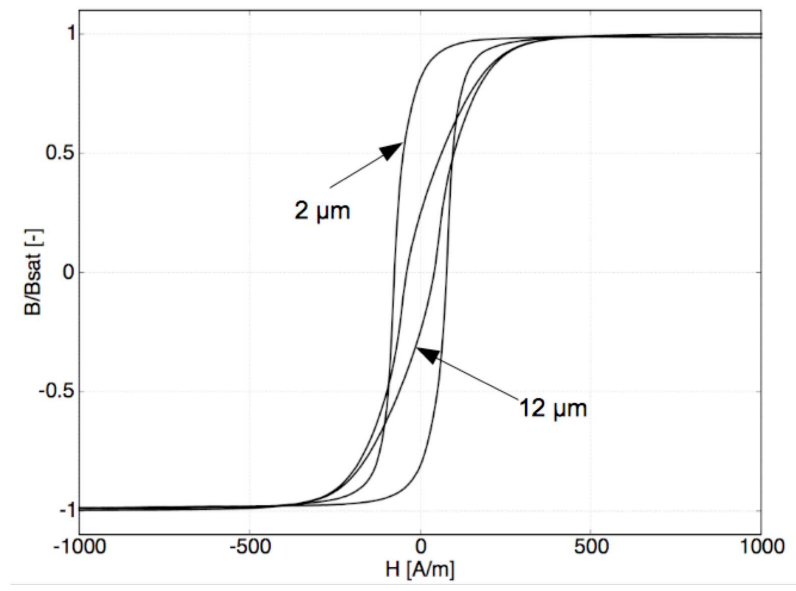

Fig. 2. Hysteresis loops of ring cores electroplated under radial field with $2 \mu \mathrm{m}$ and $12 \mu \mathrm{m}$ thickness.

A possible explanation for this phenomenon can be found in the fact that when the first part of the layer is electroplated the material is exposed only to the $720 \mathrm{kA} / \mathrm{m}$ field produced by the yoke. The following parts of the ring however are electroplated on the top of a ferromagnetic layer, which has been electroplated before. This ferromagnetic layer is therefore already magnetized by the radial field. When the new layers are electroplated their magnetic moment assumes the same direction of the underlying ferromagnetic layer, which is already saturated in radial direction. In a sense, it can be seen as a sort of magnifier of the field produced by the yoke, limited to its surface.

\section{Effect of the thickness on the noise}

We used such ring-cores as base for fluxgate sensors. We used a 350 turns excitation coil wound around the ring and fed by an excitation current $I=2.5 \mathrm{~A}_{p-p}$ at $30 \mathrm{kHz}$. The pick-up coil consisted of 250 turns without tuning. We extracted the second harmonic by mean of lock-in amplifier. We measured the noise of the sensor by inserting it in a 4-layer permalloy shielding. We consider the noise at $1 \mathrm{~Hz}$ as reference value for the noise spectrum as typically done for fluxgates. We found that the noise at $1 \mathrm{~Hz}$ rapidly decreased if the thickness was increased: it dropped from $700 \mathrm{pT} / \sqrt{ } \mathrm{Hz}$ for $2 \mu \mathrm{m}$ to $60 \mathrm{pT} / \sqrt{ } \mathrm{Hz}$ for $12 \mu \mathrm{m}$, as can be seen in Fig. 3 .

As the thickness increases to $18 \mu \mathrm{m}$ the noise slightly rises again. This indicates that for thicknesses from $2 \mu \mathrm{m}$ to $12 \mu \mathrm{m}$ the dominant phenomenon is the increment of the radial anisotropy, which reduces the noise of the sensor.

Then as we increase the thickness to $18 \mu \mathrm{m}$ the anisotropy cannot increase anymore (as we have previously seen) but the demagnetization effect still increases becoming the predominant effect which makes the noise eventually rise. A confirmation comes from the dependence 


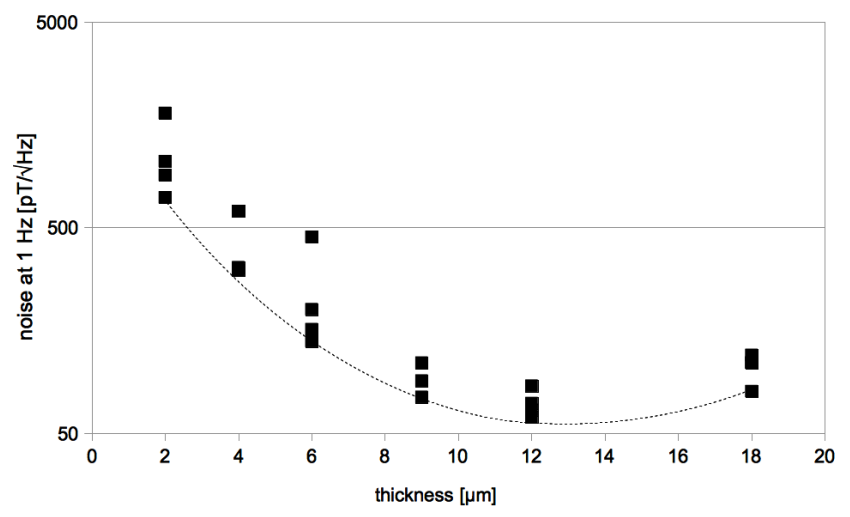

Fig. 3. Noise at $1 \mathrm{~Hz}$ of a fluxgate sensor vs. thickness of the NiFe film ring core. The dotted line shows the minimum achievable noise in case of defect-less ring.

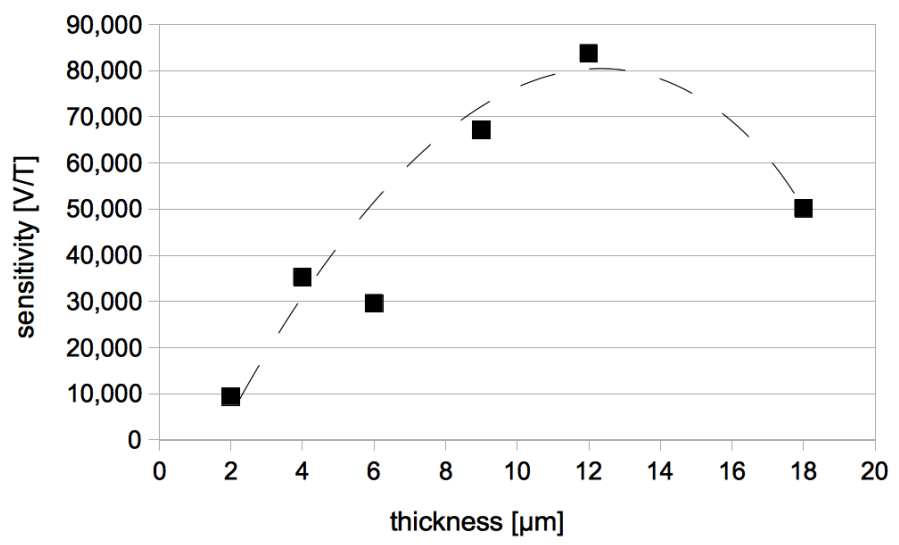

Fig. 4. Average sensitivity of the fluxgate vs. thickness of the ferromagnetic layer of the core.

of the average sensitivity of the sensor vs. the thickness, plotted in Fig. 4. As we can see at $18 \mu \mathrm{m}$ the sensitivity decreases, even if the thickness is larger, due to demagnetizing factor.

\section{Conclusions}

In this paper we have shown that the field-induced anisotropy obtained applying a strong magnetic field during the electroplating of FeNi alloy strongly depends on the thickness, up to a certain thickness (in this case $12 \mu \mathrm{m}$ ) above which does not grow anymore. For larger thickness however the demagnetizing effect plays an important role decreasing the sensitivity and therefore increasing the noise.

In conclusion, a trade-off should be always found between the need of larger thickness, which generally gives larger field-induced anisotropy, and lower thickness which is favorable against demagnetization.

\section{Acknowledgments}

This work was funded by resources provided by the Czech Science Foundation (GA ČR) under grant No. 16-10591Y.

\section{References}

[1] F. Primdahl, B. Hernando, O.V. Nielsen, J.R. Petersen, J. Phys. E Sci. Instrum. 22, 1004 (1989).

[2] M. Janošek, J. Vyhnánek, A. Zikmund, P. Butvin, B. Butvinová, Acta Phys. Pol. A 126, 104 (2014).

[3] M. Butta, P. Ripka, M. Janosek, M. Pribil, J. Appl. Phys. 117, 17A722 (2015).

[4] X.P. Li, J. Seet, J. Fan, J. Magn. Magn. Mater. 304, 111 (2006)

[5] P. Butvin, M. Janošek, P. Ripka, B. Butvinová, P. Švec Sr., M. Kuzminski, P. Švec Jr., D. Janičkovič, G. Vlasák, Sens. Actuat. A Phys. 184, 72 (2012). 